\title{
Return Current statistics in extended Josephson junctions
}

\author{
Cinzia De Leo and Giacomo Rotoli* \\ Dipartimento di Energetica and UdR INFM, Università di L'Aquila.
}

\begin{abstract}
In last years the return current, i.e., the value of the bias current where an hysteretic Josephson junction switch back to zero voltage state, have obtained interest in view of its connections with the evaluation of losses for macroscopic quantum coherence experiments and the following development of q-bit devices. Here we study carefully the problem of the return current by means of numerical simulations of associated Langevin equations in both very small and intermediate length Josephson junctions. Comparison with theoretical lifetime, fluctuations, and recent experiments are carefully carried on. The results show that the effects of finite length are important in the correct description of the return switch.
\end{abstract}

PACS: 85.25 Am, 85.25 Cp, 85.25 Hv

Keywords: Josephson junctions, fluctuations, damping.

\section{INTRODUCTION}

The return current is defined as the value of the bias current at which a Josephson junction switch back to the zero voltage state. Both the mean value of the current and the fluctuations about it have been analyzed in the past by theoretical investigations ${ }^{1,2}$ and by experiments ${ }^{3-6}$. The interest in return current is driven by its important connections with the quasi-particle losses in the junctions ${ }^{7}$. This in turn came out of great interest for the newly projected applications of Josephson junction as constitutive element of q-bit devices $^{8,9}$. On the other hand also the development of new digital Josephson devices ${ }^{10}$ and

\footnotetext{
*CORRESPONDING AUTHOR Dipartimento di Energetica and Unità di Ricerca INFM, Università di L'Aquila, Località Monteluco I67040, L'Aquila, Italy, phone +0862434331, fax +0862434303, e-mail:rotoli@ing.univaq.it,
} 
more recently developed systems as three-terminal devices ${ }^{11}$ or flip-flop devices ${ }^{12}$ push for a careful analysis of condition of junction zero-voltage reset in order to apply engineering procedures for the definition of competitive devices. In this paper we study the return current problem in the framework of RSJ model using massive numerical simulations of Josephson junction of finite extension ranging from small to intermediate length cases. Is well-known that thermal fluctuations giving arise to a finite extent of the thermal switch region. These switches can be collected in histograms and analyzed according to the known theoretical predictions as that given by Ben-Jacob et al. ${ }^{1}$ about lifetimes of running state, and variance of thermal distribution as given by Chen, Fisher and Leggett ${ }^{2}$. A comparison with existing experimental data is carried out in order to analyze the possible causes of some incongruencies between theory and experiments ${ }^{5}$.

The paper is organized as follows: in sect.I we review briefly both the deterministic and statistical theoretical framework about the return current; in sect.II we report the numerical results together with the data analysis of lifetimes and width of distributions. A brief conclusion is in sect.III.

\section{THE RETURN CURRENT}

The standard RSJ Josephson junction model is based on the view that the state of the junction is fully described by its phase variable $\varphi$ which satisfy the following equation ${ }^{7}$ :

$$
\ddot{\varphi}+\alpha_{0} \dot{\varphi}+\sin \varphi=\gamma+\gamma_{N}
$$

here times are normalized to plasma frequency $\omega_{J}=\sqrt{\frac{2 \pi I_{0}}{C \Phi_{0}}}$ with $C$ the junction capacitance, $I_{0}$ the Josephson supercurrent, $\Phi_{0}$ the flux quantum. In the above equation $\alpha_{0}=1 / \sqrt{\beta_{C}}$, with $\beta_{C}=\frac{2 \pi I_{0} C R^{2}}{\Phi_{0}}$ the Stewart-McCumber parameter that contains the bare normalized resistence $1 / \alpha_{0}$ and $\gamma$ is the normalized current bias, i.e., $\gamma=I_{b} / I_{0}$ where $I_{b}$ 
is the current feed to the junction. Finally $\gamma_{N}$ is a Gaussian current white noise, which model the effect of the thermal fluctuations.

It is worth to note that RSJ model is purely ohmic from the point of view of dissipative losses, i.e., the losses are voltage independent and reduce to the ohmic term $\alpha_{0}$. There is no gap in the RSJ model, though it can be improved using some most accurate approximation to the quasi-particle losses. On the other hand the use of such approximations is questionable ${ }^{2}$. A fully developed theory, as Quasi-Particle Tunneling (QPT) approach will be mostly suitable to describe the actual junctions. Here however we use the simplest RSJ model in order to test its limits. Moreover we are interested to compare our numerical results with experiments at $4.2 \mathrm{~K}$ (cf. Castellano et al..$^{5}$ ), so we treat the junction like a "classical" rather than a "quantum" object, i.e., we stay in the limit $\mu \ll 1$ and $\rho \ll 1$ following Chen et al. ${ }^{2}$, where $\mu=k_{B} T / I_{0} \Phi_{0}$ and $\rho=\mu / \alpha$.

A frequent misconception about the Eq.(1) is that it describe "small junctions", where the term "small" usually refers to "anything that is less than $\lambda_{L}$ ", where $\lambda_{L}$ is the socalled Josephson length $\lambda_{L}=\sqrt{\frac{\Phi_{0}}{2 \pi J_{0} \mathcal{L}}}$, with $\mathcal{L}$ the inductance per unit length of the junction and $J_{0}$ the current per unit length. Besides many actual junctions are typically $\ll \lambda_{L}$ the Eq.(1) is a correct description only for a magnetic field far from critical field of the junction. By the fact Eq.(1) describe zero length junctions with infinite critical field. So any model that would introduce magnetic field effects have to change Eq.(1) into the well-known SG equation for long Junctions:

$$
\ddot{\varphi}+\alpha_{0} \dot{\varphi}+\sin \varphi=\varphi_{x x}+\gamma+\gamma_{N}(x, t)
$$

where $\varphi_{x x}$ is the second spatial derivative of the phase. The magnetic field is set in the boudary conditions, i.e., $\varphi_{x}(0)=\varphi_{x}(l)=\eta$ where $l=L / \lambda_{L}$ is the normalized length of junction, and $\eta=\frac{2 \pi \lambda_{J} d B}{\Phi_{0}}$ the normalized magnetic field with $d$ the electric thickness of the 
junction. Here $\gamma_{N}(x)$ is a spatially distributed Gaussian white noise with autocorrelation function given by ${ }^{13}$ :

$$
<\gamma_{N}(x, t), \gamma_{N}\left(x^{\prime}, t^{\prime}\right)>=\frac{4 \pi l \alpha_{0} k_{B} T}{\Phi_{0} I_{0}} \delta\left(t-t^{\prime}\right) \delta\left(x-x^{\prime}\right)
$$

In absence of term $\gamma_{N}$ Eq.(1) is deterministic and its solutions can be expressed in terms of elliptic functions or integrated numerically. In order to grasp the essential aspects of these solutions we note that it is well-known that Eq.(1) can be thinked as the equation of the motion of a particle in the so-called washboard potential (check signs):

$$
V(\varphi)=-\gamma \varphi-\cos \varphi
$$

with a dissipative term given by $\alpha_{0} \dot{\varphi}$. The existence of a return current can be inferred by the following argument: for $\gamma \rightarrow 0$ the tilt of the potential tend to vanish so the dissipation can slow down the particle until it become unable to overcome the next potential well given by $\cos \varphi$ term. We call this value of the bias the "return current" $\gamma_{r}$. It is worth to note that the above argument make sense only for hysteretic junctions, i.e., underdamped junction with small losses $\alpha_{0} \leq 1$. On the other hand the hysteretic junction are the most promising for applications, both "classical" and "quantum", being a two-state system with very small losses. It is worth to note that bare dissipation represented by the value of $\alpha_{0}$ which enter in the Eq.(1) could not confused with "true" dissipation $\alpha$ which can due to other phenomena which are not described by Eq.(1). The effect of magnetic field, noise or junction length can change the dissipative properties: no one of these effects are present in the deterministic, i.e., $\gamma_{N}=0$, Eq.(1) above. From here we assume that relation which can be deduced on the basis of RSJ model are valid for generic, true or bare, quantities. The failure of these relation in the data analysis will indicate the limit of the model and the necessity of its improvement. This is analogue to analysis made in 
Castellano et al. ${ }^{13}$. It is possible evaluate the deterministic value of $\gamma_{r}$ which results given by the following expression ${ }^{1}$ :

$$
\gamma_{r}=\frac{4 \gamma_{c}}{\pi} \alpha
$$

where $\gamma_{c}$ is the normalized critical current. The temperature behavior of deterministic return current $\gamma_{r}$ was recently analyzed in a careful comparison with experiments by M.G.Castellano et al. ${ }^{6}$ for both RSJ and QPT models. The results show that low critical current density junctions have lower dissipation.

Adding the noise term $\gamma_{N}$ the Eq.(1) become a Langevin equation. Applying statistical procedures it is possible to evaluate the escape rate from oscillatory state ${ }^{1}$ :

$$
\Gamma=A\left(\gamma_{r}\right) \exp \left(-B\left(\gamma_{r}, \alpha\right)\right)=\omega_{J} \sqrt{\frac{\left(\gamma-\gamma_{r}\right)^{2} E_{J}}{\pi k T}} \exp \left(-\frac{\left(\gamma-\gamma_{r}\right)^{2} E_{J}}{\alpha^{2} k T}\right)
$$

where $E_{J}=\frac{\Phi_{0} I_{0}}{2 \pi}$ is the Josephson energy. As $\Gamma$ can be evaluated by experiments or also by numeric simulations the Eq.(5) can be used to fit other parameters as the temperature $T$ and the "true" value of return current $\gamma_{r}$, or, the "true" loss coefficient $\alpha$ and the "true" value of return current. According to above explained procedure fit can be used to check the consistency of the theory giving information on the parameters appearing in Eq.(5), i.e., we can assume that also for finite (but moderate) length junctions the Eq.(5) is valid, except that true parameters could be not satisfy point-like junction relations like Eq.(4).

Another independent theoretical prediction on the return current is the width of return switches distribution, which in the thermal noise limit can be written:

$$
w_{t h}=2 \sigma=\gamma_{r} G(\mu, v) \sqrt{\frac{1}{2} \mu f(T) \ln 2}
$$

where $G(\mu, v)$ is a function of the order of 1 in which $\mu$ and $v$ enter in logarithmic form and $f(T)$ is a growing function of temperature ${ }^{2}$. In Eq.(6) current interval given by $w_{t h}$ 
is normalized to maximum Josephson current. At $T=0 \mathrm{~K}$ we have $f(0)=1$, whereas at $T=4.2 \mathrm{~K} f(4.2) \simeq 10$ for the experimental data in ref.4.

\section{NUMERICAL RESULTS AND DISCUSSION}

If theory given in Ben-Jacob et al. ${ }^{1}$ is again valid in the description of finite, but short length junctions described by Eq.(2) then we can use numerical simulations of this equation to predict the true value of return current and one of two other parameters, i.e., the temperature or the loss coefficient $\alpha$. Here to make a comparison with Castellano et al. ${ }^{5}$ we set $T=4.2 \mathrm{~K}$ in Eq.s(3),(5) and evaluate the most interesting loss coefficient $\alpha$. We use the standard procedure to simulate Eq.(2) dividing the junction in $N$ discrete sections each with length $\Delta x$ so that $l=N \Delta x$. We analyze two normalized length $l=1$ and $l=0.15$ using $\Delta x=0.05$ this implies $N=20$ in the first case and $N=3$ in the second case. If we assume current density to be constant the ratio of critical currents for the two length will be $3 / 20$ this means that variances in Eq.(3) will have the same ratio.

We assume that smallest length have exactly the same critical current of junction in the experiment ${ }^{5}$. In some case we take two junction length have the same critical current, which means a different current densities. Magnetic field behavior for zero voltage escape in long junctions was treated in Castellano et al. ${ }^{13}$ where is shown that for junction normalized length below one the behavior is very similar to point-like junction until the neighbor of the critical field.

Numerical procedure is similar to that used in Castellano et al. ${ }^{13}$ for zero voltage escape. Junction is set in the resistive state and the system of ODE equivalent to Eq.(2) is integrated with a adiabatic down sweep of bias current until return switch occurs. Then the procedure is repeated until we reach a significant statistics. Normalized sweep times $1 / v$ are roughly of the order of $3 \times 10^{-6}$. 
The result of return switch sampling are plotted in the form of histograms. In Fig.1 we show typical histograms for the two junction lengths in zero magnetic field. In Fig.1a the case of smaller length for a deterministic dissipation of $\alpha_{0}=0.025$ is shown. This value of bare dissipation is about the same of the experiment ${ }^{5}$. The sample is formed by $n=5827$ return switches. We note that histogram have a well defined width, so we do not observe in the simulation the extremely small $\sigma$ found in the experiment. In Fig.1b we report the histogram for the longest junction with the same dissipation and critical current density, with $n=2123$. For the longer junction the histogram mean value and width are lower because the critical current is larger. In Fig.2a we report histograms for the same parameters of Fig.1a except that a magnetic field roughly equal to $\eta_{c} / 2$ is now imposed over the junction. We note that mean value is lower with respect to Fig.1 as can be espected because the magnetic field reduces the return current. Skewness for all histograms is between 0.7 and 0.9 .

Next step in the numerical data analysis is the fit of Eq.(5 ). We note that in this case prefactor $A\left(\gamma_{r}\right)$ in Eq.(5) is depending on "true" $\gamma_{r}$ which is unknown before the fit, anyway the dependance is weak so we can use $\gamma_{r}^{0}=\frac{4 \gamma_{c}}{\pi} \alpha_{0}$ or the histogram mean value as a first guess in the fit procedure and then iterate is until reach consistency. The fit produces two unknown parameters: the "true" return current $\gamma_{r}$, from which we can calculate the "deterministic" value of dissipation $\alpha_{d}$ deduced by Eq.(4), and the "stochastic" dissipation $\alpha_{s}$. Some fit is given in Fig.3 for two values of lentgth and low bare dissipation $\alpha_{0}$. The complete sets of data collected with the fit of Eq.(5) are reported in Table I which would be compared with Fig.7 of Castellano et al. ${ }^{5}$. Values of dissipation are given as normalized resistor $1 / \alpha$ in order to have a direct comparison with discussion of experiment. We note that in zero magnetic field just at the first guess $\gamma_{r}^{0}$ fitted "true" return current is found to 
be always within its associated error equal to its deterministic value given by Eq.(4). The same is true for "deterministic" value of dissipation $\alpha_{d}$ in zero magnetic field. This is an indication that theoretical model is again reliable for finite length junctions in zero field. When magnetic field is different from zero $1 / \alpha_{d}$ is smaller according as in the experiment, meaning that magnetic field increase the dissipation. Anyway the "stochastic" dissipation $\alpha_{s}$ is always larger than its deterministic value by a factor variable between 1.37 and 3.15. This happens systematically for each values of length, dissipation and critical currents and is also observed in the experiments where the factor was larger $(\simeq 10)$. It is also interesting that this effect is larger in small junction case than in large junction case, and with the same length is larger for small critical current junctions (cf. ref.5). This seems indicates that the observed noise reduction of dissipation is effective in very small junctions with low critical current. On the other hand the magnetic field behavior follows the deterministic case, i.e., the "stochastic" resistor $1 / \alpha_{s}$ is smaller, again implying that magnetic field increase dissipation. There is no substantial differences between two analyzed lengths, also if these are again small to produce relevant differences. Naturally on this basis we are unable to decide if dissipation increase due to magnetic field have to be assigned to a real additional dissipative process or to a variation of other parameters appearing in Eq.(5) as in the case of zero voltage escape ${ }^{13}$. It could be linked to the torque applied by the field on the pendula in the mechanical analog of the junction, this torque favors early switch of the junction to the zero voltage state, so producing an increase of the dissipation. This is a typical finite length effect which do not implies a real increase of dissipation.

In Table II we report the numerically evaluated widths. Can be seen that widths are smaller for low dissipation case and higher critical currents. In low dissipation case 
we observe an increase of the width in presence of magnetic field which was noted in the experiment ${ }^{5}$ also if the increase is moderate. This could agree with above fit analysis showing that fluctuations are enhanced in presence of a magnetic field. Anyway for higher dissipation the same does not happen and the width decrease in presence of magnetic field. Comparison with theoretical prediction given by Eq.(6) shows that numerically evaluated width is always smaller than theoretical one by a factor variable between 2 and 4 . This is similar to what happens in the experiment ${ }^{5}$. Accordingly to above fit analysis this would be expected because (apparently) numerical thermal noise reduces the dissipation. We note however that we use full expression given in ref.2, whereas in ref.4 comparison is made with $T=0$ value of $w_{t h}$ which is lower.

\section{CONCLUSION}

The indications emerging by the above numerical analysis can be resumed in two main conclusion. Firstly the numerical simulations seem to confirm what was found in the experiments because both analysis of escape by fitting Eq.(5) or width of distributions are different from theoretical prediction based on simple RSJ model. Anyway this conclusion require attention: numerical data show the tendencies found in the experiment, but not the quantitative values. Furthermore the finite length of the junctions do not alter substantially the zero magnetic field values of (deterministic) dissipation and return current. But in non zero magnetic field case could be plausible that an (apparent) increase of dissipation is related to the internal dynamic of a finite length junction.

\section{ACKNOWLEDGMENTS}

We thank M.G.Castellano, C.Cosmelli, P.Carelli, G.Filatrella and G.P.Pepe for useful discussions and suggestions. We gratefully acknowledge financial support from MURST 
COFIN2000 project "Dynamics and Thermodynamics of vortex structures in superconductive tunneling". 


\section{REFERENCES}

${ }^{1}$ E.Ben-Jacob, D.J.Bergman, B.J.Matkowsky and Z.Schuss, Phys.Rev.A26, 2805, (1982).

${ }^{2}$ Y.C.Chen, M.P.A.Fisher and A.J.Leggett, J.Appl.Phys.64, 3119, 1988.

${ }^{3}$ J.R.Kirtley, C.D.Tesche, W.J.Gallagher, A.W.Kleinsasser, R.L.Sandstrom, S.I.Raider, and M.P.A.Fisher, Phys.Rev.Lett.61, 2372, 1988.

${ }^{4}$ A.T.Johnson, C.J.Lobb, and M.Tinkham, Phys.Rev.Lett.65, 1263, 1990.

${ }^{5}$ M.G.Castellano, G.Torrioli, F.Chiarello, C.Cosmelli, and P.Carelli, J.Appl.Phys.86, 6405, 1999.

${ }^{6}$ R.Cristiano, L.Frunzio, C.Nappi, M.G.Castellano, G.Torrioli, and C.Cosmelli, J.Appl.Phys.81, 7418, 1997.

${ }^{7}$ A.Barone and G.Paterno', Physics and Applications of Josephson Effect, Wiley, New York 1982.

${ }^{8}$ M.F.Bocko, A.M.Herr, M.J.Feldman, IEEE Trans.Supercond.7, 3638, 1997.

${ }^{9}$ P.Carelli, M.G.Castellano, F.Chiarello, C.Cosmelli, R.Leoni, G.Torrioli, IEEE Trans.Supercond.11, 210, 2000.

${ }^{10}$ P.Bunyk and D.Zinoviev, IEEE Trans.Supercond.11, 529, 2000.

${ }^{11}$ G.P.Pepe, G.Ammendola, G.Peluso, A.Barone, E.Esposito, R.Monaco, N.E.Booth, Appl. Phys. Lett.77, 447, 2000.

${ }^{12}$ G.P.Pepe, G.Peluso, M.Valentino, A.Barone, L.Parlato, E.Esposito, C.Granata, M.Russo, C.De Leo, and G.Rotoli, submitted to Appl.Phys.Lett. April 2001, arXiv: cond-mat/0105342.

1312 M.G.Castellano, G.Torrioli, C.Cosmelli, A.Costantini, F.Chiarello, P.Carelli, G.Rotoli, M.Cirillo, and R.L.Kautz, Phys. Rev.B54, 15417, 1996. 


\section{FIGURE AND TABLE CAPTIONS}

Fig. 1. Histograms of return switches in zero magnetic field for junction with bare dissipation $\alpha$ equal to to 0.025 , and temperature $4.2 \mathrm{~K}$. (a) $l=0.15$ with $n=5827$ collected switches and critical current of $15 \mu A$; (b) $l=1.0$ with $n=2123$ collected switches and critical current of $100 \mu \mathrm{A}$.

Fig. 2. Histograms of return switches for a normalized magnetic field $\eta$ roughly equal to $\eta_{c} / 2$. Parameters are the same of Fig.1. a) $l=0.15$ with $n=3959$ collected switches; (b) $l=1.0$ with $n=1372$ collected switches.

Fig.3 Linear fit of Eq.(5) for the low dissipation case $\alpha=0.025$ and $l=0.15$. On the $\mathrm{y}$ axis is the logarithmic escape rate which contain also the prefactor $A\left(\gamma_{r}\right)$. Temperature is set to $4.2 \mathrm{~K}$. (a) $\eta=0$; (b) $\eta \simeq \eta_{c} / 2$.

Table I Results of linear fit of Eq.(5) for numerical simulations data. Temperature is fixed to $4.2 \mathrm{~K}$. True return current is given normalized to maximum critical current.

Table II Width of distributions for numerical data. Temperature is fixed to $4.2 \mathrm{~K}$. Distribution widths are normalized to maximum critical current. $w_{t h}$ is the theoretical width calculated using Eq.(6). 
Table I

\begin{tabular}{cccccccc}
\hline$l$ & $n$ & $I_{c} \mu A$ & $\eta$ & $\gamma_{r}^{t}$ & $1 / \alpha_{0}$ & $1 / \alpha_{d}$ & $1 / \alpha_{s}$ \\
\hline 0.15 & 5827 & 15 & 0 & $0.0332 \pm 0.0019$ & 40 & $38.35 \pm 2.18$ & $122.65 \pm 3.40$ \\
\hline 0.15 & 3959 & 15 & 21 & $0.0290 \pm 0.0014$ & 40 & $27.83 \pm 1.39$ & $84.77 \pm 1.69$ \\
\hline 0.15 & 5360 & 15 & 0 & $0.3112 \pm 0.0129$ & 4 & $4.09 \pm 0.17$ & $10.98 \pm 0.22$ \\
\hline 0.15 & 4046 & 15 & 21 & $0.2727 \pm 0.0163$ & 4 & $2.97 \pm 0.18$ & $7.41 \pm 0.23$ \\
\hline 1.0 & 2123 & 100 & 0 & $0.0309 \pm 0.0036$ & 40 & $41.25 \pm 4.86$ & $62.00 \pm 3.55$ \\
\hline 1.0 & 1372 & 100 & 3.14 & $0.0260 \pm 0.0051$ & 40 & $31.19 \pm 5.92$ & $41.95 \pm 4.08$ \\
\hline 1.0 & 1984 & 15 & 3.14 & $0.0276 \pm 0.0043$ & 40 & $29.38 \pm 4.59$ & $92.54 \pm 7.07$ \\
\hline 1.0 & 1849 & 100 & 0 & $0.3081 \pm 0.0215$ & 4 & $4.13 \pm 0.28$ & $8.37 \pm 0.29$ \\
\hline 1.0 & 4361 & 15 & 0 & $0.3101 \pm 0.0186$ & 4 & $4.10 \pm 0.25$ & $10.41 \pm 0.32$ \\
\hline 1.0 & 1059 & 100 & 3.14 & $0.2485 \pm 0.0086$ & 4 & $3.26 \pm 0.11$ & $6.16 \pm 0.10$ \\
\hline
\end{tabular}

Table II

\begin{tabular}{ccccccc}
\hline$l$ & $n$ & $I_{c} \mu A$ & $\eta$ & $1 / \alpha_{0} w \cdot 10^{3}$ & $w_{t h} \cdot 10^{3}$ \\
\hline 0.15 & 5827 & 15 & 0 & 40 & 0.577 & 1.251 \\
\hline 0.15 & 3959 & 15 & 21 & 40 & 0.615 & 1.721 \\
\hline 0.15 & 5360 & 15 & 0 & 4 & 4.765 & 11.73 \\
\hline 0.15 & 4046 & 15 & 21 & 4 & 4.297 & 16.18 \\
\hline 1.0 & 2123 & 100 & 0 & 40 & 0.279 & 0.518 \\
\hline 1.0 & 1372 & 100 & 3.14 & 40 & 0.307 & 0.729 \\
\hline 1.0 & 1984 & 15 & 3.14 & 40 & 0.562 & 1.638 \\
\hline 1.0 & 1849 & 100 & 0 & 4 & 2.237 & 5.168 \\
\hline 1.0 & 4361 & 15 & 0 & 4 & 5.051 & 11.68 \\
\hline 1.0 & 1059 & 100 & 3.14 & 4 & 1.975 & 6.566 \\
\hline
\end{tabular}




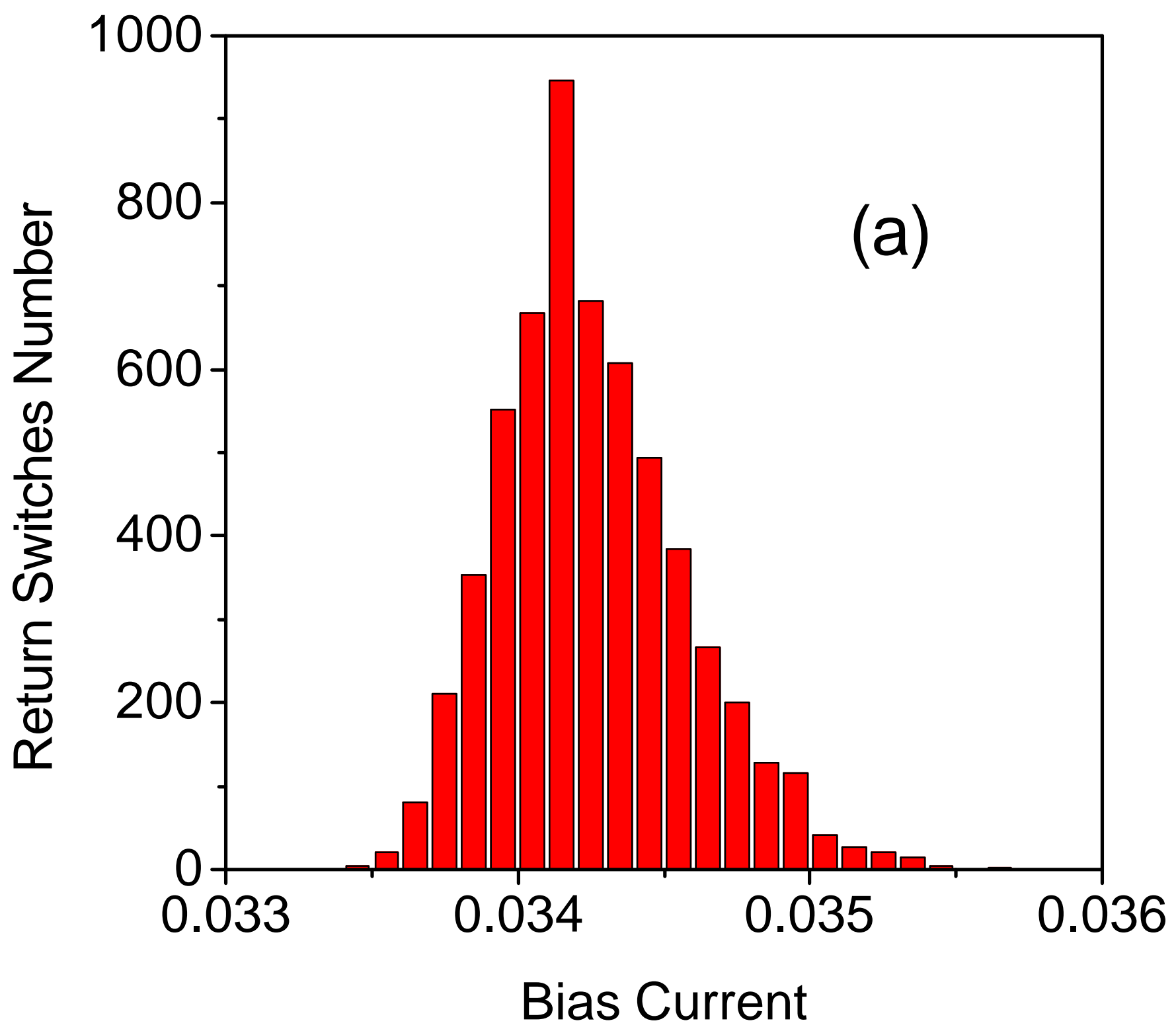




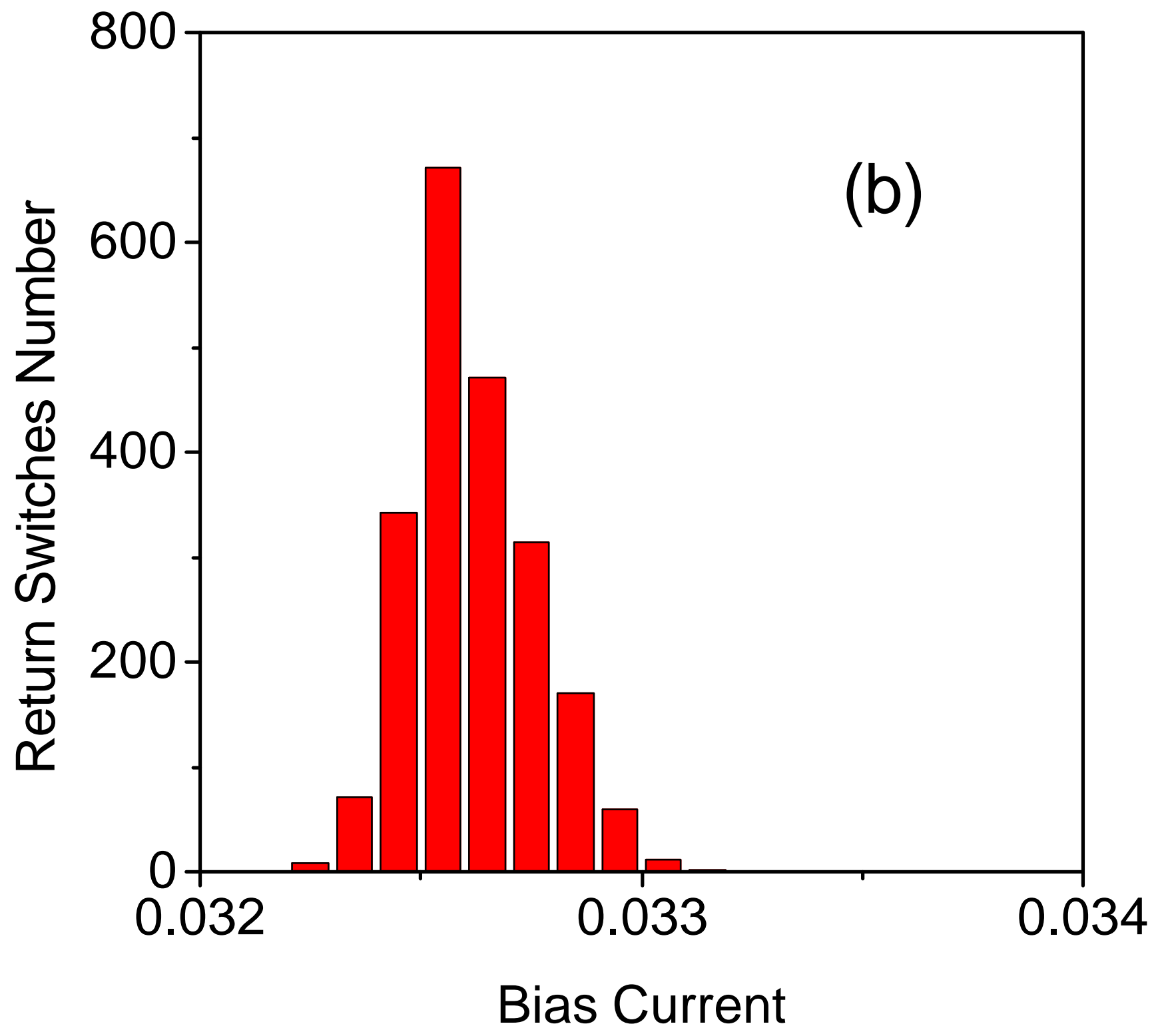




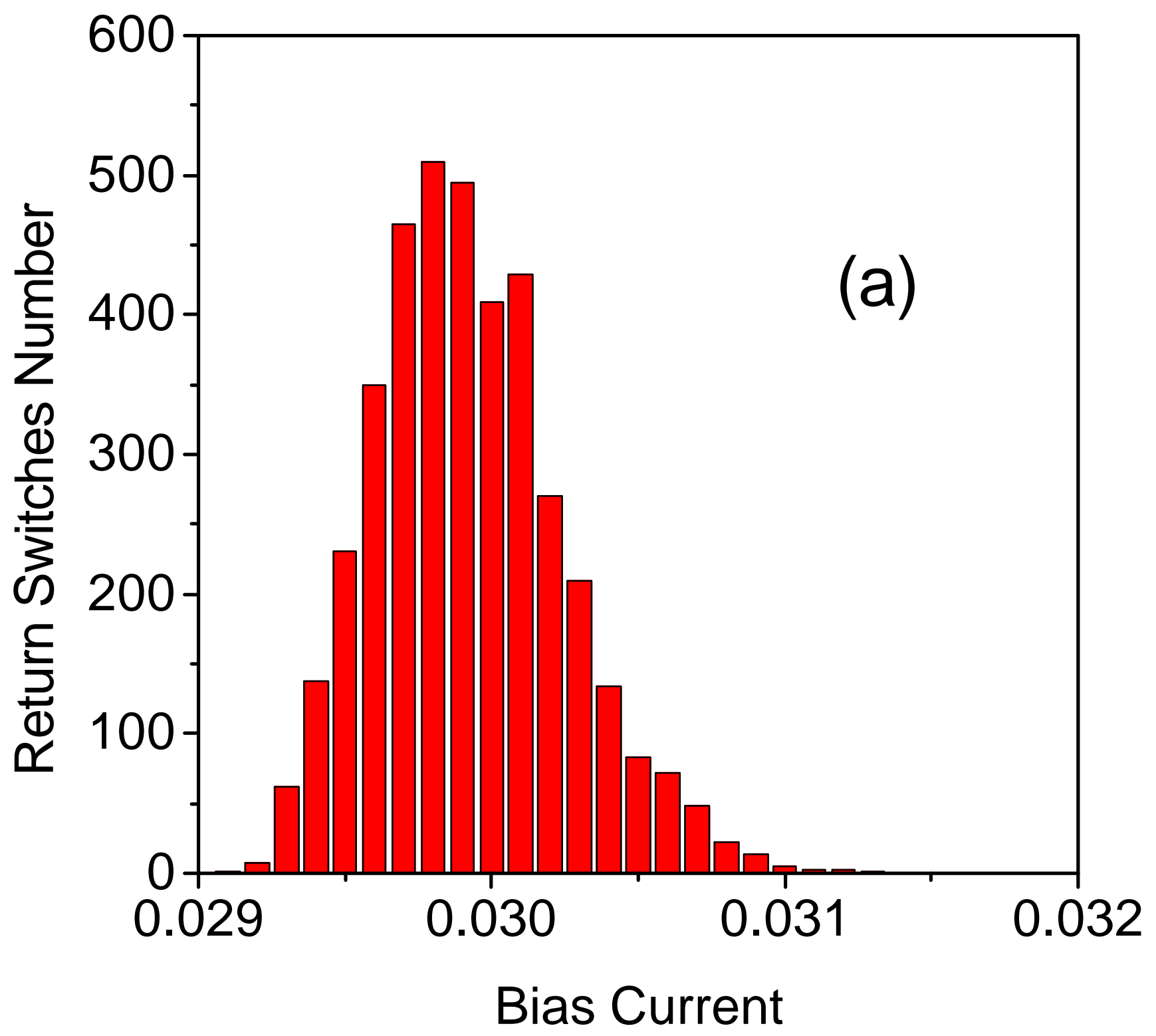




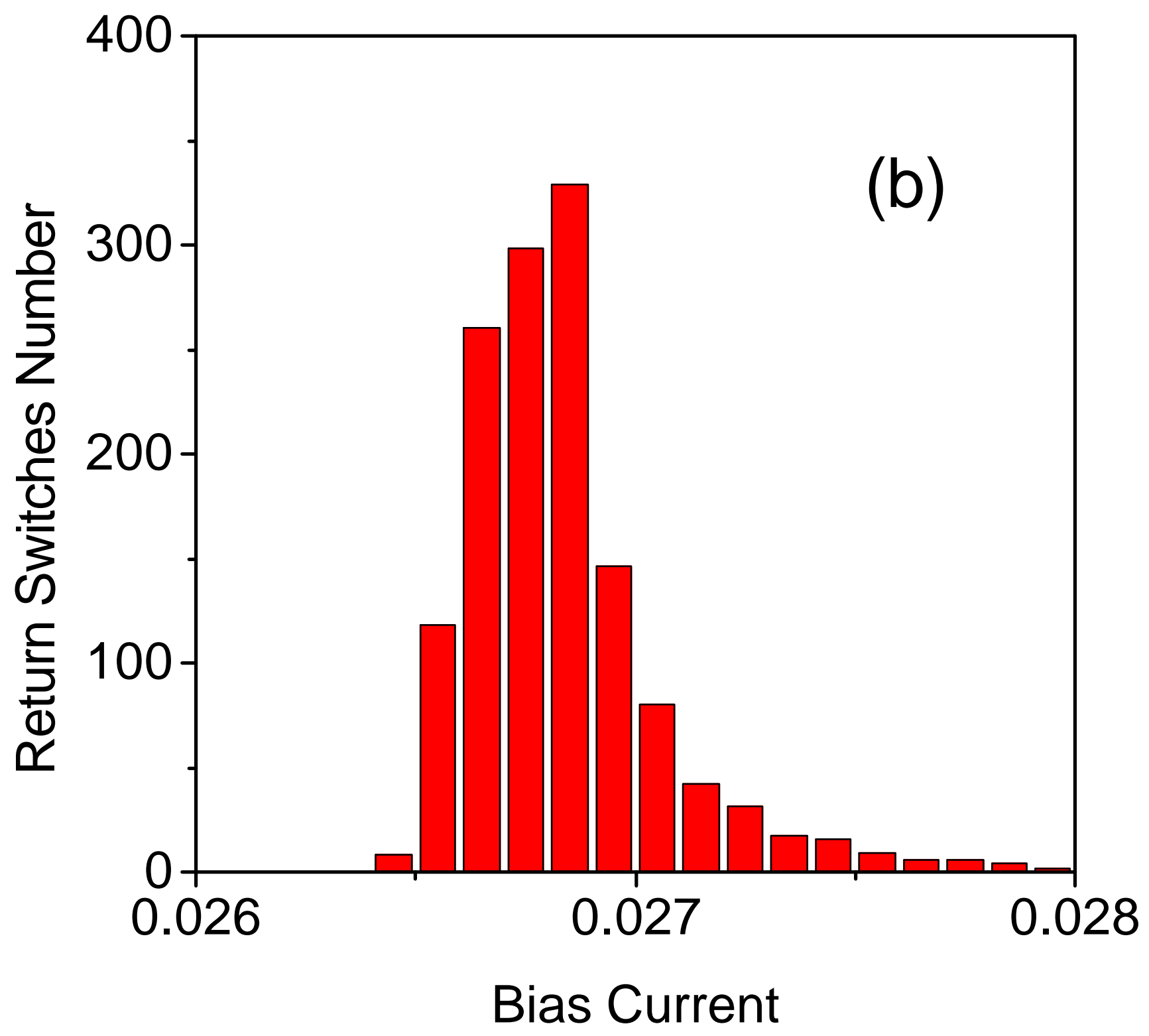




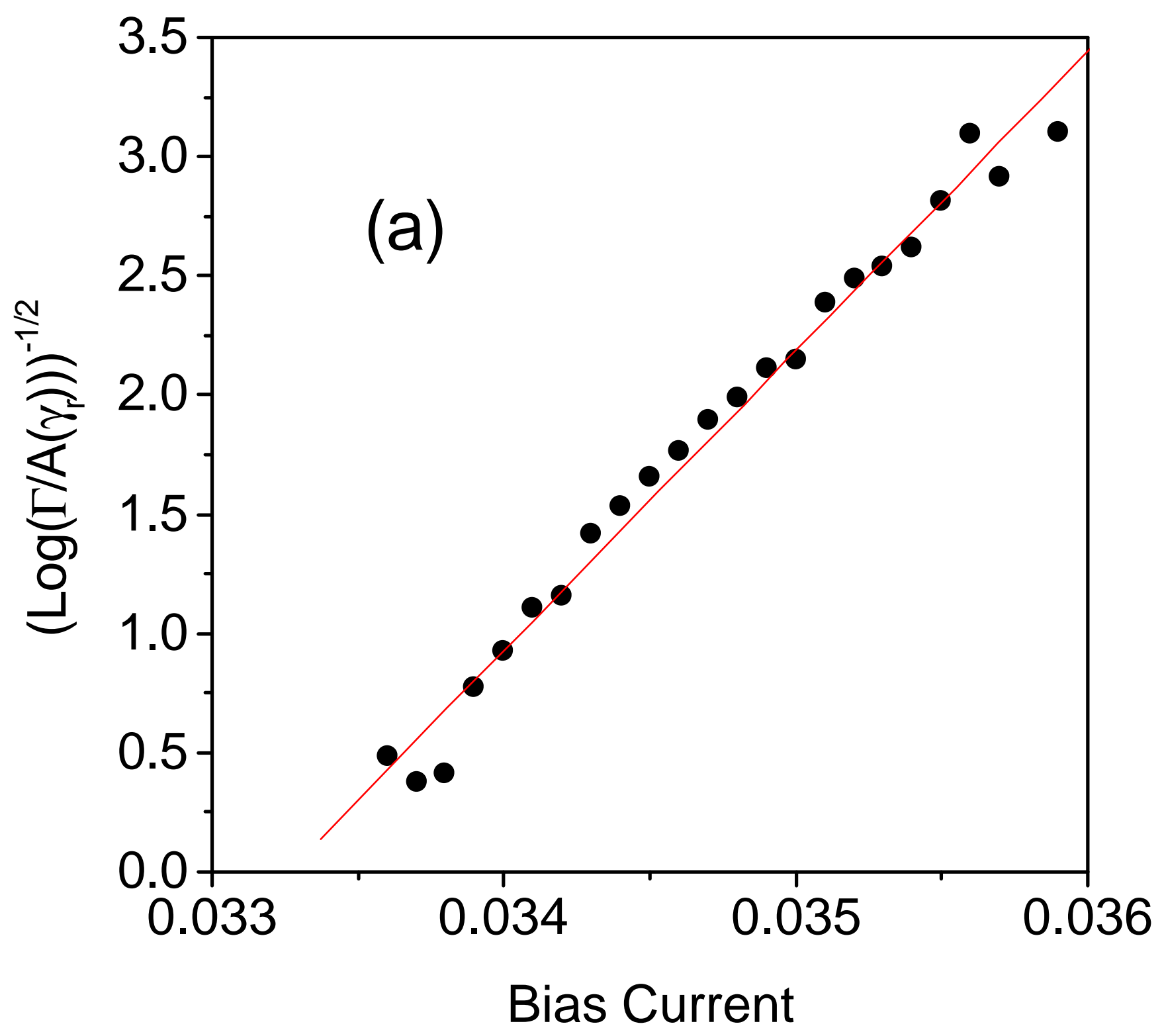




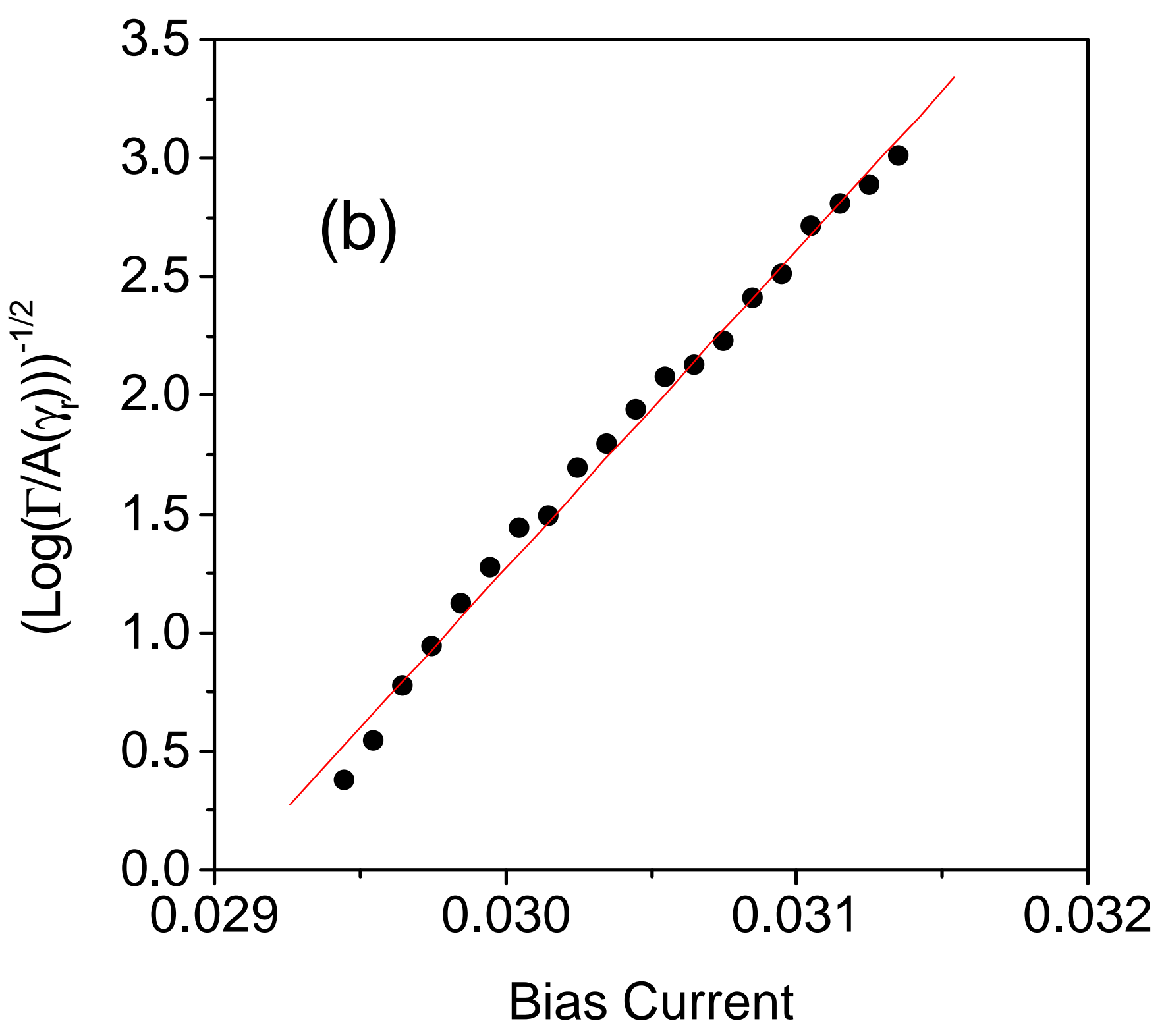

